\title{
Erratum to: A Learning-Free Approach to Whole Spine Vertebra Localization in MRI
}

\author{
Marko Rak ${ }^{(\bowtie)}$ and Klaus-Dietz Tönnies \\ Department of Simulation and Graphics, Otto von Guericke University, \\ Magdeburg, Germany \\ rak@isg.cs.ovgu.de
}

\section{Erratum to:}

Chapter 33 in: S. Ourselin et al. (Eds.)

Medical Image Computing and Computer-Assisted Intervention - MICCAI 2016

DOI: $10.1007 / 978-3-319-46723-8 \_33$

The published version of this article shows major alignment issues in Figure 2. In particular, the overlayed vertebrae candidates (the vector graphics elements) and the underlying spine images (the raster graphics) are misaligned noticeably. Presumably, these errors were introduced accidentally by a wrong conversion between graphics file formats during the publication process. We kindly thank Springer for the immediate support and correction. 